\title{
Una taxonomía del problema de distribución de planta por procesos y sus métodos de solución
}

\section{RESUMEN}

El problema de distribución de planta se puede generalizar como un caso de optimización combinatoria intensamente estudiado y por lo tanto su naturaleza es un NP-difícil. El núcleo del problema originalmente introducido como el Planeamiento Sistemático de la Distribución, un procedimiento multi-criterio y relativamente simple, se ha extendido a contextos mayores, pasando por la formulación como un modelo matemático según la naturaleza de los talleres de trabajo. Dada la complejidad computacional de los cálculos se han desarrollado heurísticas, metaheurísticas y otros para obtener soluciones aproximadas. El objetivo de éste estudio es analizar investigaciones recientes en los problemas de distribución de planta dentro del contexto industrial en orden de clasificar y comparar la representación del problema, métodos de solución, restricciones y la función objetivo usado. El informe cubre más de 30 artículos revisados sobre el tema. Particular atención es puesta sobre recientes publicaciones que han aparecido desde 2008-2013 para focalizar en los nuevos avances en el Estado del Arte.

Palabras clave: problemas de distribución de planta, modelamiento matemático, estado del arte, heurísticas, metaheuristicas

A TAXONOMY OF THEPROBLEM OF DISTRIBUTION OF PLANT PROCESSES AND METHODS OF SOLUTION

\section{ABSTRACT}

The plant layout problem can be generalized as a case intensively studied combinatorial optimization and therefore its nature is a NP- hard. The core of the problem originally introduced as the Systematic Distribution Planning, a multi -criteria and relatively simple, has been extended to larger contexts through the formulation as a mathematical model based on the nature of the workshops. Given the computational complexity of the calculations have been developed heuristics ,metaheuristics and others to obtain approximate solutions. The aim of this study is to analyze recent research on plant distribution problems within the industrial context by classifying and comparing the representation of the problem, solution methods, constraints and the objective function used . The report covers more than 30 peer-reviewed articles on the subject. Particular attention is placed on recent publications that have appeared from 2008 - 2013 to focus on new developments in the State of the Art.

Keywords: facility layout problem, mathematical modelling, state-of-the-art, heuristics, metaheuristics

\section{INTRODUCCIÓN}

El problema de distribución de planta es la determinación del arreglo físico más eficiente de un número de instalaciones que interactúan en un sistema de producción con miras de encontrar uno o más objetivos. Una instalación es cualquier entidad que ocupa espacio: por ejemplo, una persona o grupo de personas, un área de recepción de clientes, la ventanilla de un cajero, una máquina, una estación de trabajo, un departamento, un pasillo o un cuarto de almacenamiento (Krajewsky et al., 2008). En general, la distribución se planea para minimizar determinado criterio: distancias recorridas, tiempo total de fabricación, flujo y costo del manejo de materiales, retrasos o reprocesamientos y manejos físicos. También se presentan situaciones en las cuales la distribución se diseña para maximizar un criterio: espacio de utilización, flexibilidad, eficiencia, satisfacción y seguridad de los trabajadores.

Una buena distribución de estaciones de trabajo contribuye en la eficiencia global de las operaciones y puede reducir los gastos operativos hasta un 50\% (Tompkins et al., 1996). Koopmans y Beckmann (1957) son los primeros en modelar los Problemas de Distribución de Planta (Facility Layout Problems-FLP) de área igual como un problema de asignamiento cuadrático (Quadratic Assignment ProblemQAP), donde se minimiza los costos del manejo de materiales, que causa ubicar cada una de las máquinas en cada una de las localizaciones posibles. Desde entonces muchos investigadores han destacado la importancia de los QAP y su relevancia en los problemas de distribución de planta de áreas iguales.

En los sistemas de manufactura realmente los departamentos tienen áreas desiguales. Armour y Buffa (1963) propusieron un método de intercambio en parejas para los FLP de área desigual sin restricciones de forma. Tong (1991) afronta este problema asumiendo que los departamentos tienen forma rectangular y los ubica en bahías. La estructura de bahía flexible es definida como una representación continua permitiendo a los departamentos

\footnotetext{
* UNMSM, FQIQ. E-mail: maxleyvac2@hotmail.com

** UNMSM, FISI. E-mail: dms research@yahoo.com

*** UNMSM, FII. E-mail: jsalasb $@$ hotmail.com
} 
a ser localizados sólo en bahías paralelas con anchos diferentes. Wong y Komarudin (2010) le dan relevancia especial a esta representación. Garcia et al. (2013) representa el cromosoma del layout en la estructura de bahía flexible, para distribuir instalaciones desiguales en el área de una planta habitualmente rectangular de $\mathrm{H} \times \mathrm{V}$.

Tam (1992a,b) implementó otro tipo de representación continua. la estructura del árbol de corte (STS), para modelar las instalaciones que tienen forma rectangular y geometría flexible, lo que significa que el ratio entre la altura y la anchura del área asignada al departamento puede variar dentro de ciertos márgenes.

Nawaz et al. (2013) en su método propuesto para los FLPs multiobjetivos también usa la estructura del árbol. Para un layout con $\mathbf{n}$ instalaciones, el árbol rebanado consiste de $\mathbf{n}$ hojas y $\mathbf{n}-\mathbf{1}$ nodos internos, donde cada hoja representa una instalación y cada nodo interno contiene información acerca de la dirección del corte, ya sea horizontal o vertical.

\section{PROBLEMAS DE DISTRIBUCIÓN DE PLANTA}

Los intentos por establecer una metodología que permitiera afrontar el problema de distribución de planta de manera ordenada comienzan en la década de los 50 del siglo pasado. Sin embargo, es Muther (1961), el primero en desarrollar un procedimiento verdaderamente sistemático, el Planeamiento Sistemático de la Distribución (Systematic Layout Planning - SLP) que establece una metodología aplicable a la resolución del problema independientemente de su naturaleza.

Los métodos precedentes al SLP son simples e incompletos si se considera el problema de distribución en planta con un mínimo de su complejidad, sin embargo, es necesario contemplarlas desde su propio contexto. Por otra parte, los métodos inmediatamente posteriores al SLP son en muchos casos variantes de éste más o menos ampliados, mejorados y con diferentes métodos de formulación.

\subsection{Procedimiento del secuenciamiento simple de instalaciones}

El método desarrollado por Buffa (1955) puede considerarse un predecesor del SLP, pudiendo establecerse con éste muchas similitudes. El análisis de la secuencia de operaciones desarrolla un buen esquema para la distribución física, al analizar de manera gráfica el flujo de productos que se mueven entre los departamentos. El grafo debe tratar de minimizar el número de cruzamientos de las líneas de transporte de materiales y que los departamentos con mayor flujo entre ellos queden situados lo más próximo posible.

\subsection{Planeamiento Sistemático de la Distribución como un proceso precursor}

El planeamiento fue desarrollado por Richard Muther (1961] como un procedimiento sistemático multicriterio y relativamente simple, para la resolución de problemas de distribución en planta de diversa naturaleza. El método es aplicable a problemas de distribución en instalaciones industriales, locales comerciales, almacenes y otros.

El Planeamiento Sistemático de la Distribución (Systematic Layout Planning-SLP) se asienta sobre la base de la información $(P, Q, R, S, T)$ referente al problema a resolver para, a través de un proceso de diez etapas, obtener una distribución válida como solución al problema planteado.

\section{NATURALEZA DE LOS TALLERES DE TRA- BAJO}

En la terminología del análisis del proceso productivo se entiende por taller de trabajo (workshop) como la ubicación física donde se realiza un conjunto particular de tareas. Laura García H. (2011), luego de la revisión exhaustiva de la literatura ha encontrado que en la problemática de distribución de planta, influyen muchos factores según la naturaleza de los talleres de trabajo. Se ha esquematizado toda la problemática en función de dichos factores y formas en la Figura 1.

Siendo los factores más predominantes: sistema de manejo de materiales, características del sistema productivo, dispositivos adicionales, formas $y$ dimensiones de las instalaciones, número de niveles (pisos) a considerar y el horizonte del planeamiento. A continuación una breve descripción de cada uno de ellos.

\subsection{Sistema de manejo de materiales}

Uno de los factores importantes para el estudio de la disposición de planta es el factor material, pues de su tipo, variedad y cantidad dependen por lo general el tipo de sistema de producción, el cual nos llevará a un determinado tipo de distribución de planta (Díaz et al., 2008). Por otro lado, las características físicas y químicas del material determinarán los sistemas de acarreo y almacenamiento que se deberán aplicar en la planta. 


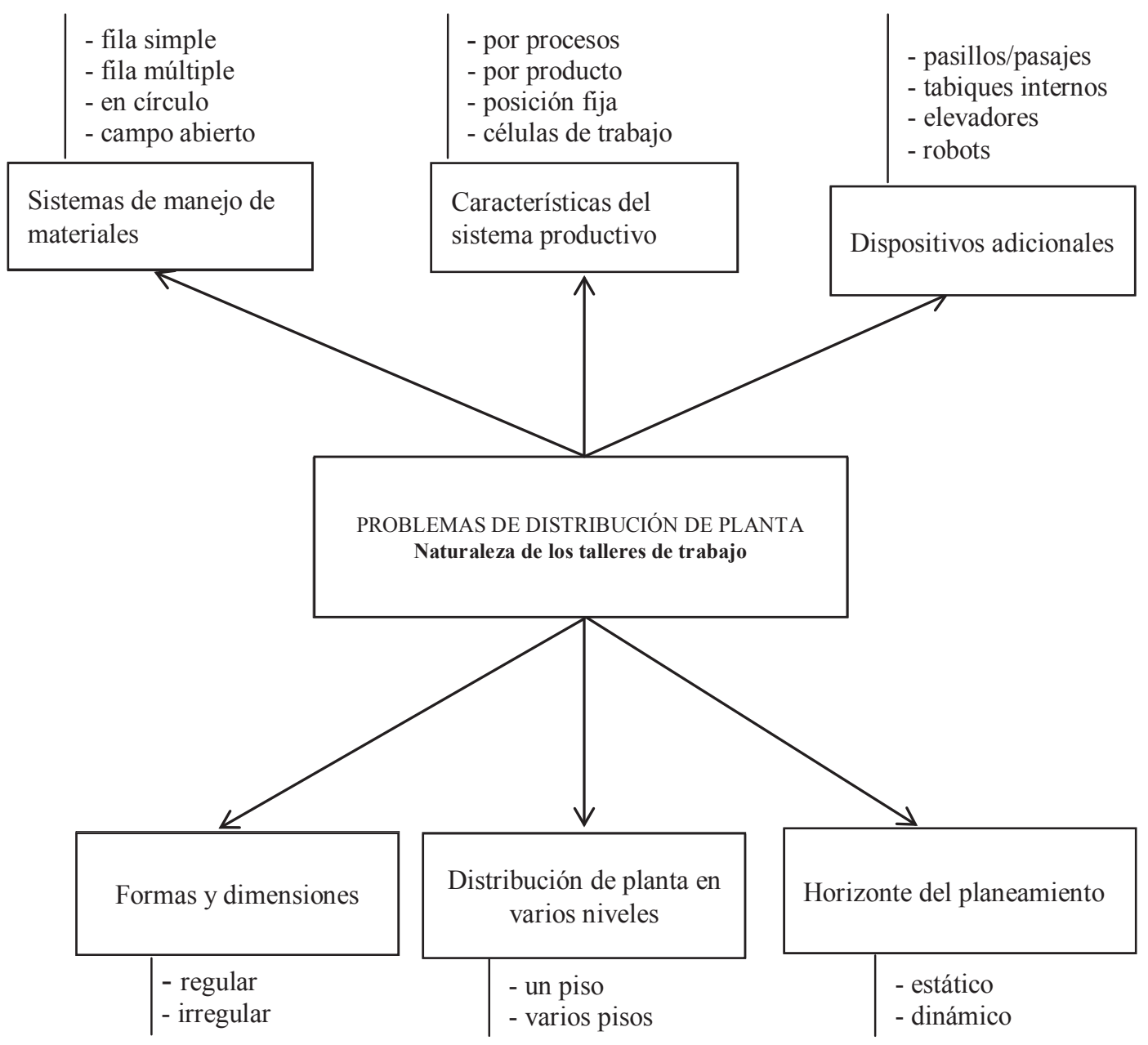

Figura 1. Naturaleza de los talleres de trabajo y su influencia en los FLP (García 2011).

\subsection{Características del sistema productivo}

Hay diferentes distribuciones de planta dependiendo del volumen y variedad de productos. Los formatos según los cuales se arreglan los departamentos están definidos por el patrón general del flujo de trabajo (Chase et al., 2009), existen tres tipos básicos: la distribución por proceso, la distribución por producto y la distribución por posición fija.

Una distribución por proceso es un formato según el cual los departamentos o estaciones de trabajo involucrados en el proceso de producción se agrupan por el tipo de función que realizan, tales como soldadura, tratamiento térmico, pintura y otros. La distribución por proceso, frente a otros tipos de disposiciones, ofrece una gran flexibilidad en cuanto a la variedad de productos, siendo su eficiencia dependiente del tamaño del lote producido.

Este tipo de distribución es típica en la industria farmacéutica en la producción de cápsulas, tabletas y tabletas especiales. También en las líneas de producción de chocolates y helados.

Una distribución por producto se adopta cuando se fabrica un producto estandarizado, por lo común en gran volumen. Cada una de las unidades en producción requiere de la misma secuencia de operaciones de principio a fin. Si se considera en exclusiva la secuencia de operaciones, la distribución es relativamente sencilla, pues se trata de colocar cada operación tan cerca como sea posible de su predecesora. Este sistema permite reducir tiempos de fabricación es decir posee una elevada eficiencia.

Las líneas de producción para los zapatos, las plantas de envasados de líquidos o embotelladoras y las empresas de ensamblaje automotriz son todas distribuciones por producto.

Una distribución por posición fija, es en la que el producto permanece en una posición fija de principio a fin, ejemplo un barco de alto tonelaje. 


\subsection{Dispositivos adicionales}

Los problemas de distribución de planta pueden incorporar dispositivos adicionales, tales como pasadizos, tabiquerías, elevadores, montacargas, robots, vehículos guiados automáticamente entre otros.

\subsection{Formas y dimensiones}

Hay dos formas comunes de las instalaciones. Por un lado la forma regular, la cual es usualmente una instalación rectangular. Por el otro lado tenemos la forma irregular, la cual es usualmente un polígono cubriendo un ángulo de 270 grados.

\subsection{Distribución de planta en varios niveles}

La ubicación de las instalaciones en una planta manufacturera pueden abarcar varios pisos o niveles. En este tipo de configuración, el material no sólo fluye horizontalmente en un piso, si no también de un piso a otro en dirección vertical.

\subsection{Horizonte del planeamiento}

Si la demanda del producto cambia en períodos cortos de tiempo, es necesario que la planta manufacturera sea flexible y capaz de adaptarse rápidamente a la nueva configuración. Si el requerimiento de los productos es constante en un periodo de tiempo largo será recomendable una distribución de planta estática, tal como ocurre en las refinerías de petróleo.

\section{REPRESENTACIÓN DEL LAYOUT}

Modelar la solución a un problema de distribución de planta implica definir la representación de las diferentes instalaciones en el área de la localización. Como indican los autores Diego-Más (2006), García (2011) y García et al. (2013) los problemas de distribución de los departamentos pueden modelarse usando varias representaciones, las mismas que pueden clasificarse y agruparse dentro de tres categorías. Al primer tipo se le denomina representación discreta, al segundo representación continua y al tercero representación cartesiana, tal como se esquematiza en la Figura 2.

\subsection{Representación discreta}

Las técnicas que formulan el problema de la distribución de instalaciones bajo modelos discretos, parten de una subdivisión del área de planta en una rejilla de cuadrículas de igual área, tal como se puede observar en la fila 4 de la Tabla 1. Las instalaciones serán situadas en la localización asignándoles el número suficiente de subdominios para cubrir sus necesidades espaciales. Si las instalaciones tienen dimensiones iguales y formas regulares, tenemos el problema de ubicar $\mathbf{n}$ instalaciones dentro de $\mathbf{m}$ posiciones, a la que se denomina el Problema de Asignamiento Cuadrático (QAP). Otros autores han aplicado la representación discreta, por ejemplo Buffa et al. (1963) y Tate \& Smith (1995).

Sin embargo si las dimensiones son desiguales es necesario adoptar otra estructura adicional. En ese sentido Balakrishnan et al. (2003) usó otra estructura adicional llamado Curva de Llenado de Espacio (SFC).

\subsection{Representación continua}

De manera general los modelos continuos construyen las diferentes distribuciones de las

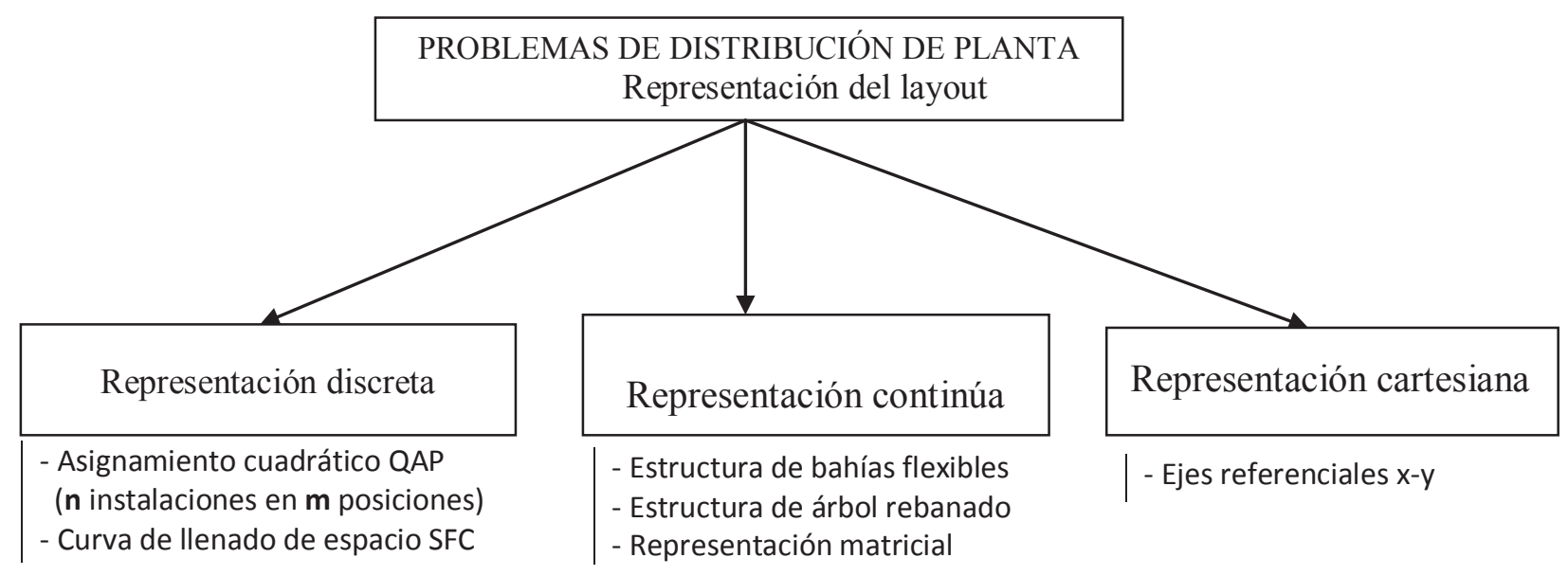

Figura 2. Técnicas de representación del Layout. 
instalaciones en el área de la planta mediante el corte recursivo del mismo. Las instalaciones tienen forma rectangular y geométrica flexible, lo que significa que el ratio entre la altura y la anchura del área asignada a la actividad puede variar dentro de ciertos márgenes (restricciones geométricas).

En la representación continua, básicamente existen dos posibles estructuras, las que emplean árboles de corte (Slicing Tree Structure STS) y los modelos de bahías flexibles (Flexible Bay Structure FBS).

\section{Estructura de bahías flexibles}

La generación de layouts mediante bahías flexibles fue propuesto por Tong (1991), permite la distribución de instalaciones rectangulares de área desigual en áreas habitualmente rectangulares. La localización es dividido en una dirección determinada en "bahías" de anchura variable, tal como se aprecia en la fila 6 de la Tabla 1. Dentro de cada una de estas bahías se coloca un número también variable de instalaciones que poseerán igual anchura, y altura proporcional al área requerida por cada una.

Actualmente está recibiendo mayor atención de los investigadores, tal como Wong y Komarudin (2010).

\section{Estructura del árbol rebanado}

Los árboles de corte como forma de representación de la distribución en planta, aparecen en el inicio de la década del 90 tal como se recoge en Tam (1992a, b). Usa la representación del árbol para describir un layout, donde cada hoja representa una instalación y cada nudo interno es el operador del rebanado. Estos operadores pueden ser 'b' corte abajo, 'u' corte arriba, 'r' corte a la derecha y 'l' corte a la izquierda, tal como se puede observar en la fila 5 de la Tabla 1.

\subsection{Representación cartesiana}

La distribución de instalaciones bajo este esquema de representación parte del origen (0) del sistema de coordenadas cartesianas. Los departamentos adoptan generalmente formas rectangulares 0 cuadradas delimitadas por su ancho $\left(a_{\mathrm{i}}\right)$ y su alto $\left(b_{i}\right)$. La ubicación de una instalación i queda definida mediante las coordenadas de su centro de gravedad $\left(\mathbf{x}_{i}, \mathbf{y}_{\mathrm{i}}\right)$ respecto al origen. Todos los modelos analíticos usan esta representación.

\section{MÉTODOS DE SOLUCIÓN}

Según la literatura revisada de los autores José A. Diego-Más (Diego-Más 2006), Laura García H. (García 2011), Hamdy A. Taha (Taha 2012) y el Handbook of Combinatorial Optimization; se puede ordenar y clasificar los diferentes métodos de solución en función de la técnica empleada para la resolución, la misma que se presenta en la Figura 3.

\subsection{Métodos exactos}

Tratan de obtener la mejor solución global al problema. Son aplicados fundamentalmente a formulaciones del problema como de asignamiento

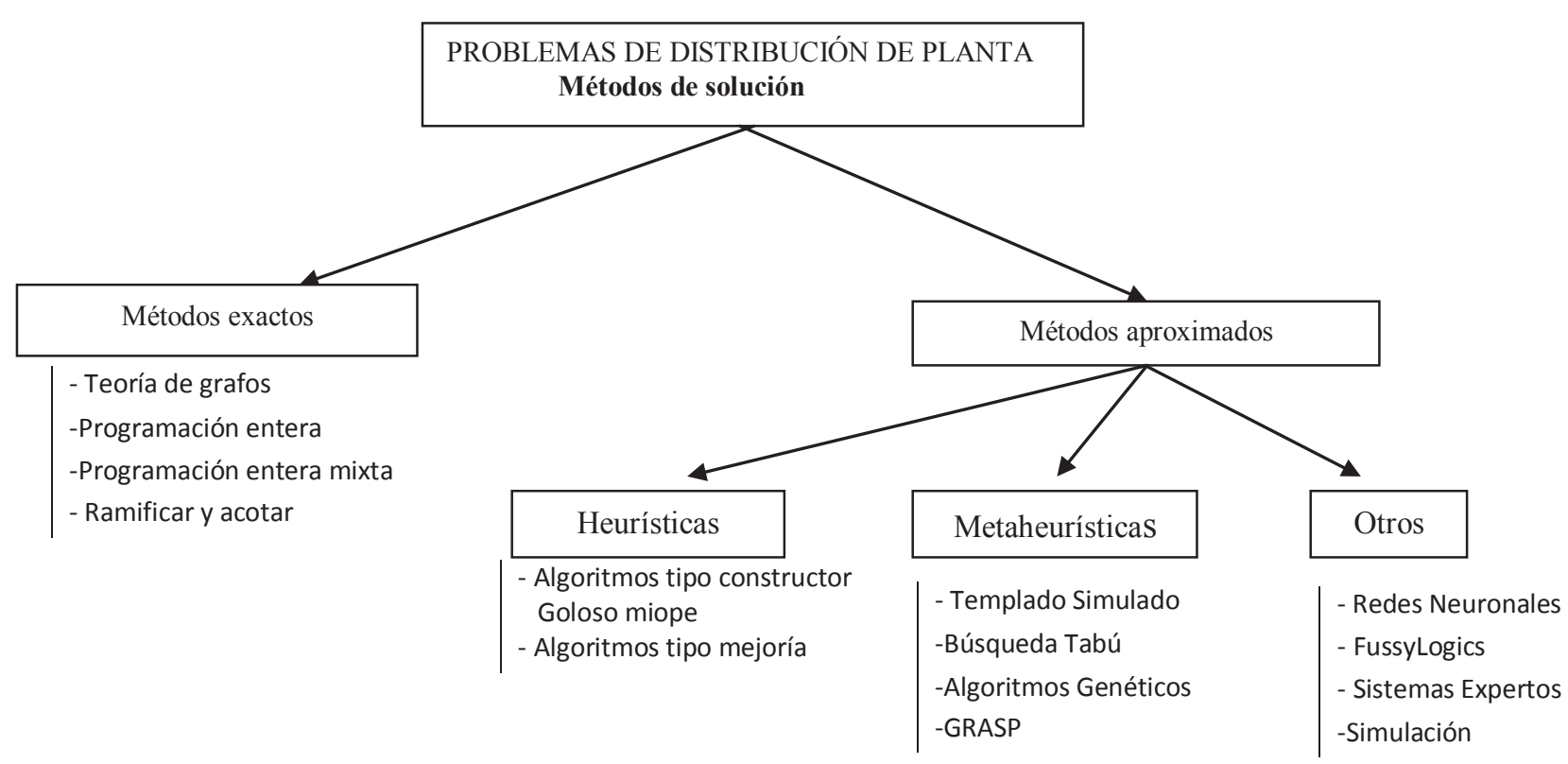

Figura 3. Métodos de solución de los Layout. 
cuadrático (Koopmans et al., 1957). En general las instalaciones tienen igual área (monoárea) y las posiciones de asignación están fijadas a priori. Algunos de estos métodos son estrictamente enumerativos, es decir, tratan de evaluar todas las posibles soluciones al problema, lo cuál, dado el carácter complejo del mismo, los hace absolutamente ineficientes y poco operativos para problemas de cierta envergadura. Pertenecen a este grupo los métodos de ramificar y acotar, programación entera y teoría de grafos.

\section{Algoritmo de ramificar y acotar}

Las primeras referencias a este tipo de algoritmos aplicables a problemas cuadráticos de asignación se pueden encontrar en Gilmore (1962). En Loiola et al., (2004) se realiza una enumeración exhaustiva de publicaciones que emplean algoritmos de branch and bound. Este tipo de algoritmos parten de una solución inicial generalmente obtenida mediante algún algoritmo heurístico.

Las técnicas branch and bound han evolucionado mucho en los últimos años, Gilmore resolvió en 1962 un problema cuadrático de asignación de tamaño 8 (Gilmore, 1962), en el año 2000 Anstreicher y sus colaboradores (Anstreicher et al., 2002) fueron capaces de resolver el problema tipo de tamaño 30 de Nugent (nug30).

\section{Algoritmo MILP: problemas de programación entera mixta}

Montreuil (1990) introduce la programación entera mixta para los UA-FLP, cuyo objetivo está basado en el producto del costo del manejo de materiales y la distancia rectilínea entre los centroides de los departamentos. Aunque este es un método potente, solamente problemas con 6 o menos instalaciones pueden ser resueltos óptimamente (Meller \& Gau, 1996).

Gan Xu \& Lazaros G. Papageorgiou (2009) desarrolló un modelo de programación lineal entera mixta para un número pequeño de variables y restricciones, en una planta de producción de óxido de etileno. Dado un conjunto de ítems de equipos con sus dimensiones y costos de conexión entre ellos, el problema consiste en determinar la localización de cada ítem de equipo (en coordenadas y orientación), con tal de minimizar el costo total de conexión y sujeto a restrcciones de no superposición y pertenencia al dominio del área de la planta, ver fila 8 de la Tabla 1.

\subsection{Métodos aproximados}

Para la mayoría de los problemas de distribución en planta es imposible encontrar procedimientos exactos de solución que operen en tiempos realistas y con tecnologías asequibles. Como alternativa surgen los procedimientos heurísticos.

\section{Heurísticas}

Existen diversos intentos de dar una definición de los procedimientos heurísticos; por ejemplo en (Zanakis et al., 1981) se puede leer que son:

... procedimientos simples, a menudo basados en el sentido común, que tienden a ofrecer una buena solución (aunque no necesariamente la óptima) a problemas difíciles, de un modo fácil y rápido.

En el presente trabajo se emplea como criterio de clasificación la forma de generar las soluciones, agrupándose los métodos en: métodos constructivos, métodos de mejora y métodos híbridos.

\section{Heurística: Algoritmos tipo mejoría}

Los métodos de mejoría parten de una solución inicial del problema, a partir de la cual realizando modificaciones sistemáticas, obtienen diferentes soluciones mejoradas.

A este tipo de algoritmos pertenece el CRAFT (Buffa et al., 1964) que viene a ser el método tipo mejoría más antiguo. Comienza determinando el costo total de una distribución inicial dada. En seguida realiza intercambios de dos o tres formas de los centroides de instalaciones no fijas que son también de áreas iguales o adyacentes en el layout. Para cada intercambio CRAFT calcula y estima la reducción en el costo y escoge el intercambio con la reducción estimada más grande. El proceso de intercambio continua hasta que ya no exista reducción estimada haciendo los intercambios de dos o tres formas. Hicks \& Cowen (1976) criticaron el procedimiento de intercambio porque puede llevar a las instalaciones a tener formas irregulares. De este tipo de métodos, CRAFT (Computarized Relative Allocation of Facilities Technique) es quizá el más conocido, muchos otros métodos son variaciones realizadas sobre la base de éste.

SPACECRAFT (Johnson, 1982) es otro método en una fase derivado de otro que emplea modelos bidimensionales, en este caso el CRAFT, pero ampliado a edificios de varios niveles.

WinQSB (Chang 2000), este programa resuelve el problema de distribución funcional usando el mismo algoritmo de CRAFT, la cual es una heurística para mejorar la distribución inicial por intercambio de departamentos. Maneja varios parámetros adicionales. 
Además pertenecen a este grupo la mayor parte de las técnicas basadas en búsqueda tabú, recocido simulado y algoritmos genéticos.

\section{Heurística: Algoritmos tipo constructor}

De manera general los métodos constructivos generan los layouts escogiendo una tras otra las instalaciones a distribuir, y colocándolas en determinadas posiciones del dominio de ubicación.

A este tipo de algoritmos pertenece CORELAP, que fué desarrollado por Lee \& Moore (1967). Ellos usan las relaciones de cercanía de cada instalación para determinar una distribución. Bajo este criterio, la instalación con la calificación de cercanía más alta es seleccionado y asignado al centro del área de la planta, para orientar la distribución de los departamentos restantes. Las instalaciones subsiguientes son adicionados al Layout dependiendo de sus relaciones a las instalaciones ya localizadas.

ALDEP ha sido desarrollado por Seehof \& Evans (1967). Este método trabaja en la siguiente forma, primero una instalación es seleccionada aleatoriamente y es ubicado en el rincón izquierdo superior del layout. La siguiente instalación escogida para ser localizado es uno que tiene la calificación de cercanía mayor o igual a un relación de cercanía especificado por el usuario, con una selección aleatoria para la primera instalación. Este método puede manejar distribuciones en edificios de varios niveles.

De manera general la mayor parte de los métodos basados en técnicas de corte o teoría de grafos y algunos que hacen uso de la lógica difusa pertenecen a este grupo.

\section{Metaheurísticas}

La teoría de la complejidad nos indica que, para los problemas de distribución en planta de cierta envergadura y con un número suficiente de restricciones, es muy improbable encontrar métodos exactos de resolución.

Osman (Osman, 1995) proporciona una definición acertada de metahuristica:

Dentro de la clase denominada metahuristicas se incluye todos aquellos procedimientos que en un proceso iterativo; guían a una heurística subordinada combinando inteligentemente diferentes conceptos tomados de analogías de la naturaleza, y exploran el espacio de soluciones utilizando estrategias de aprendizaje para estructurar la información, con el objeto de encontrar eficientemente soluciones cercanas al óptimo.

En el análisis bibliográfico realizado por Loiola et al., (2004) sobre las publicaciones dedicadas al problemas cuadrático de asignación se observa que el número de aportaciones que emplean técnicas metaheurísticas duplica a los que emplean métodos heurísticos o exactos, tal como se muestra en la Figura 4.

\section{Metaheurística: Templado Simulado}

El algoritmo del Simulated Annealing se fundamenta en la analogía con el proceso de enfriamiento lento de los metales sólidos, donde la temperatura del material es variado en orden de cambiar sus propiedades físicas. Por analogía este proceso es aplicado para resolver problemas de optimización. Así en cada iteración, algunos vecinos son evaluados con cierta probabilidad en orden de

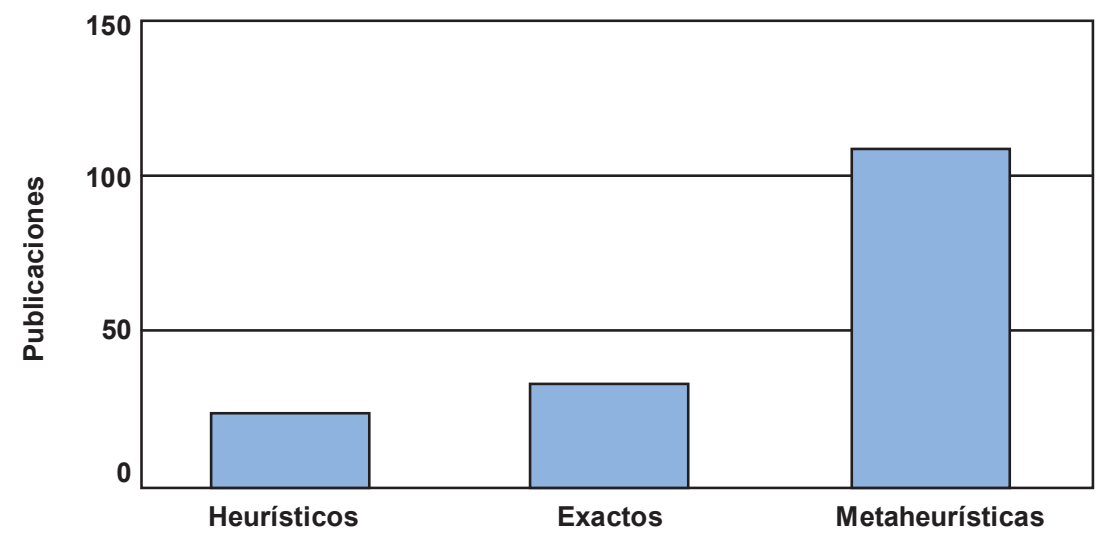

Figura 4. Número de publicaciones dedicadas al QAP. 
decidir si cambian a un nuevo estado o permanecen en el actual. El Templado Simulado ha sido aplicado por muchos autores para los FLP. Así Tam (1992a) también usó esta técnica para resolver los UA-FLP, el desarrollo LOGIC, Layout Optimization using Guillotine-Induced Cut. En orden de representar un Layout usó la representación STS. Este algoritmo aplica el TS en orden de encontrar una mejor distribución por intercambios de dos formas de los operadores de ramificación.

\section{Metaheurística: Búsqueda Tabú}

Esta metaheuristica es un método de optimización que usa un sistema de memorias estructuradas para mejorar el proceso de un método de búsqueda local. El método de Búsqueda Tabú (Tabú Search) trabaja en la siguiente forma: cuando una solución potencial es encontrada, se marca como un Tabú, y el Algoritmo no explora esta solución encontrada. Este método ha sido aplicado a FLPs por muchos autores. Así Hasan \& Osman (1995) uso esta técnica con funciones de relajamiento desarrollados para obtener soluciones cercanas al óptimo. McKendall \& Jaramillo (2006) también aplicaron esta técnica a los problemas de distribución de planta dinámica, ellos compararon sus resultados cono otros tomados desde la literatura y concluyeron que su método obtiene buenas soluciones.

\section{Metaheurística: Algoritmos Genéticos}

Los Algoritmos Genéticos plantean los problemas complejos de búsqueda y optimización bajo un enfoque evolutivo basado inicialmente y a grandes rasgos, en la teoría evolucionista del desarrollo de las especies naturales elaborada por Darwin (Goldberg, 1999). La computación evolutiva puede ser clasificada en tres categorías principales: los algoritmos genéticos (Genetic Algorithm), las estrategias evolutivas (EE) y la programación evolutiva (PE). Las diferencias fundamentales entre cada una de las técnicas radican en los tipos de alteraciones que se realizan sobre las soluciones para obtener nuevos individuos, y en los métodos empleados para la selección de los supervivientes y los reproductores.

Los AGs se han transformado en los métodos más populares para resolver los FLPs (Pierreval et al., 2003). Por esta razón hay muchos artículos que aplican los AGs para resolver FLPs tales como Tam (1992b) y Wu \& Ji (2008).

La representación del cromosoma con dos segmentos para los FLPs en la presencia de rutas de procesamiento alternativo es propuesto por Maghsud Solimanpur (2010), tal como se puede apreciar en la fila 11 de la Tabla 1.
Laura García H. (García 2011) presenta una nueva estructura de representación que consta de una matriz de 3 filas por $\mathbf{n}$ columnas, ver fila 10 de la Tabla 1. Amir Sadrzadeh (Sadrzadeh 2012) implementa una forma de codificar el cromosoma en base a relaciones de cercanía para los problemas de distribución en filas múltiples, ver fila 12 de la Tabla 1.

En las técnicas propuestas por los autores previos para los FLPs siempre se ha abordado teniendo en cuenta criterios cuantificables, Laura García et al., (García et al. 2013) presenta un Algoritmo Genético Interactivo (IGA) para evaluar adicionalmente los criterios cualitativos (ver fila 14 de la Tabla 1).

\section{Otros}

Adicionando nuevas máquinas en la planta y/o introduciendo nuevos productos puede convertirse la distribución actual en un caso ineficiente. Tales cambios pueden causar incrementos significativos en el transporte de materiales entre máquinas, disminuyendo la productividad del proceso. Para prevenir estas anomalías, una alternativa son los modelos de simulación en la computadora para los problemas de distribución de planta.

\section{CONCLUSIONES}

Todas las heurísticas de mejoría incluyendo el CRAFT como el más antiguo y popular, que parten de una distribución inicial de planta no garantizan una solución óptima. El hecho de comenzar con una solución razonablemente buena es más probable que producirá una solución final de menor costo, pero esto no sucederá siempre. Por ejemplo en el caso del Test Data de Armour \& Buffa (1963), el costo de un layout inicial escogida al azar es 10.164 y con las sucesivas mejoras se reduce el costo a 7.862 obteniendo una eficiencia del $23 \%$; pero ésta comparación no es respecto al óptimo.

Los siguientes Autores: Diego-Más (2007), García (2011), Aiello et al. (2013) y García et al. (2013) toman como un Test Data precursor el caso de estudio de 20 departamentos de Armour \& Buffa (1963).

Los algoritmos computarizados básicamente se clasifican según la naturaleza de la función objetivo. El primer grupo trata de minimizar la distancia recorrida por el flujo de materiales a través de las instalaciones, pertenecen a ésta familia CRAFT, SPACECRAFT, MÚLTIPLE y WinQSB. Estas aplicaciones se orientan hacia los FLPs del sector manufacturas y requieren un layout inicial para hacer la mejoría. 
El segundo grupo trata de maximizar la calificación total de cercanía (closeness value) para todos los departamentos, siempre cumpliendo con las características requeridas del edificio, pertenecen a éste grupo CORELAP, BLOCPLAN y ALDEP. Estas aplicaciones básicamente se orientan hacia los FLPs del sector servicios y se inicia con un área de piso vacío y luego procede a ubicar los departamentos, empezando con la instalación que tiene la calificaión de cercanía más alta.
Finalmente se presenta un resumen cronológico de los métodos de representación del layout, respecto a la propuesta de los autores, la misma que se indica en la Tabla 1. En la columna 1 se indica el tipo de representación y el Autor, en la columna 2 está la codificación de la representación y en la columna 4 se indica (n) el tamaño del problema que el Autor maneja en su caso de estudio. En la Figura 2 están detalladas las técnicas de representación del Layout.

Tabla 1. Métodos de representación del Layout

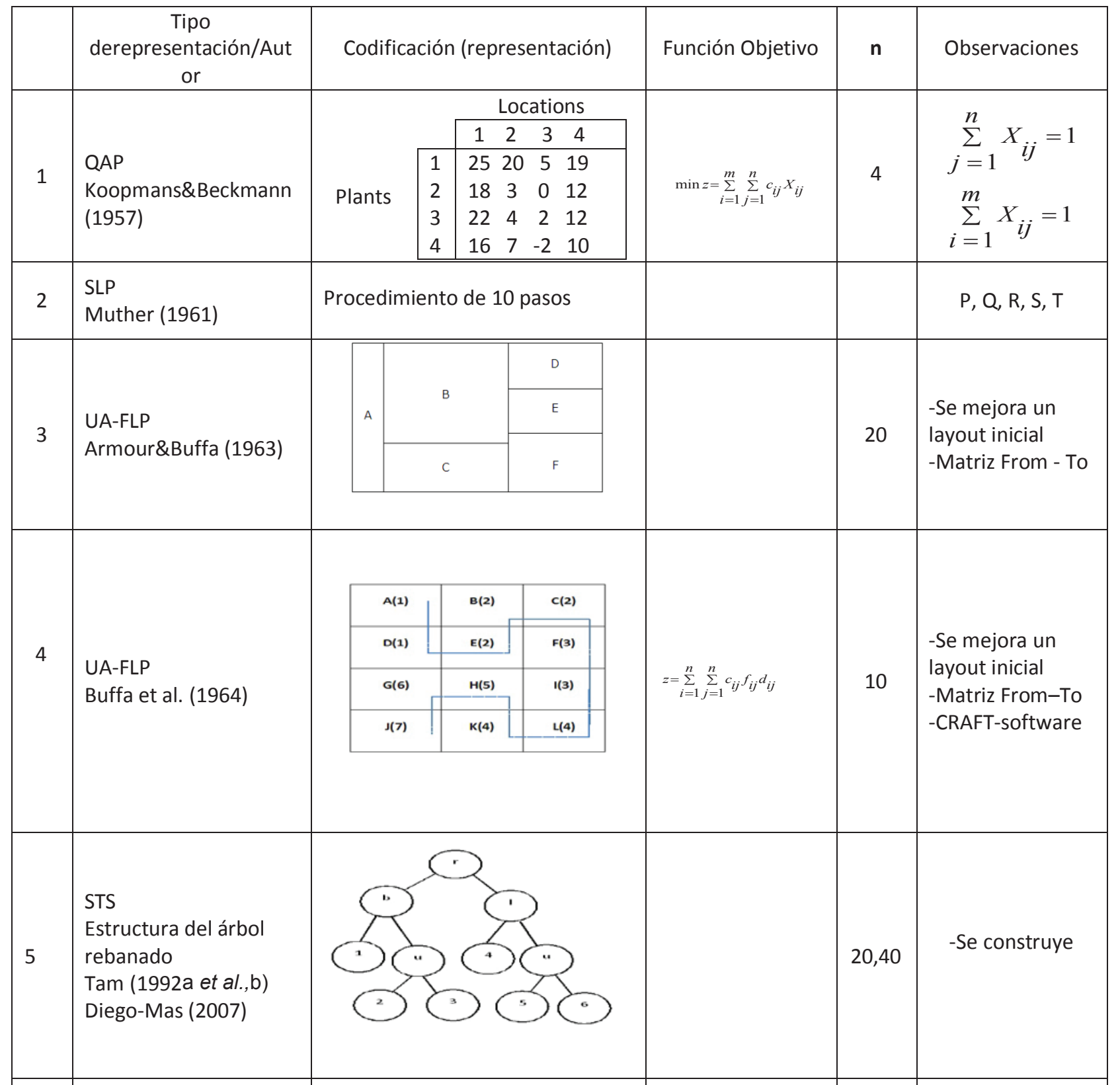




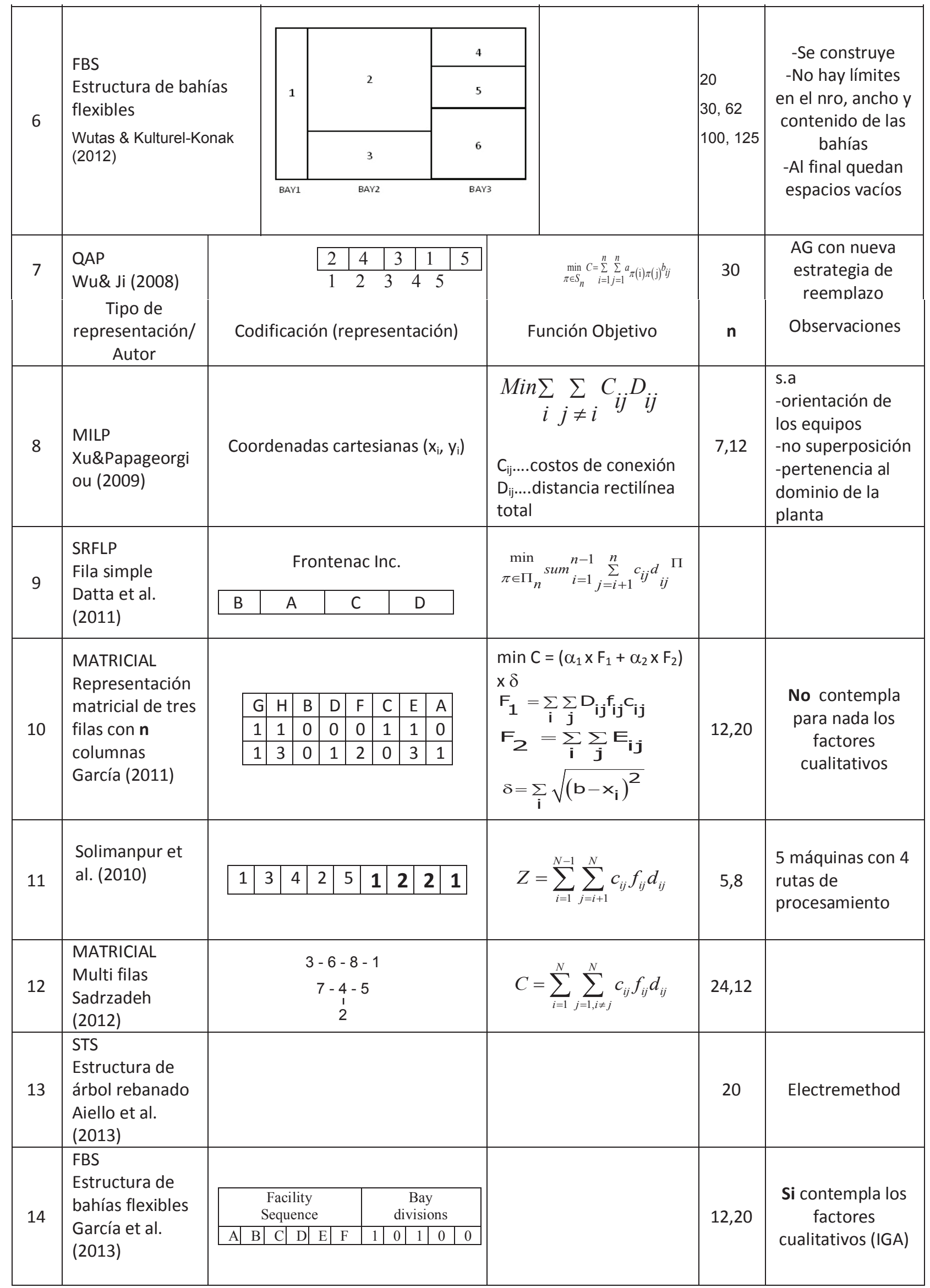




\section{REFERENCIAS BIBLIOGRÁFICAS}

[1] Adam E. y Ebert R. (1991). Administración de la Producción y las Operaciones, Prentice-Hall Hispanoamericana.

[2] Ahuja R. K., Orlin J. B. \& TiwariA. (2000). Agreedy genetic algorithm for the quadratic assignment problem, Computers and Operations Research, 27, pp. 917-934.

[3] Aiello G., La Scalia G. \& Enea M. (2013). Anon dominated ranking Multi Objective Genetic Algorithm and electre method for unequal area facility layout problems, Expert Systems with Applications 40 4812-4819.

[4] Anstreicher K.M., Brixiu, N.W., Linderoth, J., y Goux, P. (2002). "Solving large quadratic assignment problems on computational grids", Mathematical Programming, 91, pp. 563-588.

[5] Armour G.C. \&Buffa E.S. (1963). A heuristic algorithm and simulation approach to relative location of facilities, Management Science, 9, 294-309.

[6] Balakrishnan J., Cheng C.H. \& Wong K.F., (2003). FACOPT: A User FriendlyFACility Layout OPTimization System, Computers \& Operations Research,Vol. 30, pp. 1625-1641.

[7] Buffa E.S., Sequence Analysis for Functional Layouts, (March-April 1955), Journal of Industrial Engineering, Vol. 6, N. ${ }^{\circ}$ 2, pp. 12-25.

[8] Buffa E.S., Armour G.C. \&Vollman T.E., (MarchApril 1964), Allocating Facilities with CRAFT, Harvard Business Review, Vol. 42, N. ${ }^{\circ} 2$, pp. 136-159.

[9] Chase R., Aquilano N. y Jacobs R. (2009). Administración de la Producción y Operaciones para una ventaja competitiva, décima edición, McGraw-Hill.

[10] Datta D., Amaral A. \& Figueira J., (2011), Single row facility layout problem using a permutationbased genetic algorithm, European Journal of Operational Research, 213 388-394.

[11] Díaz B., Jarufe B. y Noriega M.T. (2008). Disposición de planta, segunda edición, Fondo Editorial Universidad de Lima Perú.

[12] Diego-Más J. A. (2006). Optimización de la distribución en planta de instalaciones industriales mediante algoritmos genéticos, Universidad Politécnica de Valencia, Tesis Doctoral, España.

[13] Francis, RichardL. \& White, John A. (1974). Facilities Layout and Location, Prentice Hall, Englewood Cliffs, NJ.
[14] Gaither N. \& Frazier G. (2000). Administración de Producción y Operaciones, International Thomson Editores.

[15] García H. L. (2011). Genetic Approaches for the Unequal Area Facility Layout Problem, Universidad de Córdoba, Tesis Doctoral, España.

[16] García-Hernandez L., Pierreval H., Salas-Morera L. y Arauzo-Axofra A. (2013). Handling qualitative aspects in Unequal Area Facility Layout Problem: An Interactive Genetic Algorithm, Applied Soft Computing, 13, 1718-1727.

[17] Gilmore P.C. (1962). "Optimal and suboptimal algorithms for the quadratic assignments problem", SIAM Journal on applied mathematics, 10, pp. 305-313.

[18] Goldberg D. E. (1999). Genetic Algorithms in Search, Optimization and Machine Learning, Addison-Wesley, Boston, MA, USA.

[19] Hasan M. \& Osman (1995). Local Search Algorithms for the Maximal Planar Layout Problem. International Transactions in Operational Research, Vol. 2, pp. 89-106.

[20] Hicks P.E. \& Cowen, T.E. (1976). CRAFT-M for layout Rearrangement, Industrial Engineering, Vol. 8, pp. 30-35.

[21] Johnson R.V. (1982). SPACECRAFT for multifloor layout planning, Management Science, Vol. 28, N. ${ }^{\circ} 4$, pp. 407-417.

[22] Koopmans T.C. \& Beckmann M. (Jan. 1957). Assignment Problems and the Location of Economic Activities, Econometrica, Vol. 25, N. ${ }^{\circ}$ 1, 53-76.

[23] Krajewski L.J., Ritzman L.P. \& Malhotra M.K. (2008). Administración de Operaciones Procesos y cadena de valor, Ed. Pearson Educación, México.

[24] Lee R., \& Moore J. M. (1967). CORELAPcomputerized relationship layout planning. The Journal of Industrial Engineering, Vol. 18, pp. 195-200.

[25] Loiola E.M., Abreu, N.M.M, Boaventura-Netto, P,O., Hahn, P., y Querido, T., "An analytical survey for the quadratic assignment problem", documento electrònico disponible en: http:// www.seas.upenn.edu/qaplib/QAP_Survey_04. pdf\#search='boaventura\%20net to,fecha de último acceso: 20/12/2013.

[26] Mckendall A.R. \& Jaramillo J.R. (2006). A Tabu Search Heuristic for the Dynamic Space Allocation Problem, Computers \& Operations Research. Vol. 33, pp. 768-789. 
[27] Meller R.D. \& Gau K.Y. (1996). The facility Layout Problem: recent and emerging trends and perspectives. Journal of Manufacturing Systems, Vol. 15, N. ${ }^{\circ}$ 5, pp. 351-366.

[28] Meller R.D., Chen W. y Sherali H.D. (2007). Applying the Secuence - pair Representation to Optimal Facility Layout Problems, Operations Research Letters, Vol. 35, N. ${ }^{\circ}$ 5, pp. 651-659.

[29] Montreuil, B. (1990). A Modelling Framework for Integrating Layout Design and Flow Network Design. Proceedings of the Material Handling Research Colloqium. Pp. 43-58.

[30] Muther R., (1961), Systematic LayoutPlanning, Industrial Education Institute, Boston.

[31] Nawaz R.K.S., Glette K., Nizam K.K., Hovin M. Torresen J. (2013). Adaptive variable neighborhood search for solving multi-objetive facility layout problems with unequal area facilities, Swarm and Evolutionary Computation, 8, 1-12.

[32] Noori H. y Radford R. (1997). Administración de Operaciones y Producción, Calidad total y respuesta sensible rápida, McGraw-Hill.

[33] Nugent C.E., Vollman T.E., \& Ruml J. (1968). An experimental comparison of techniques for the assignment of facilities to locations, Operations Research, 16, pp. 150-173.

[34] Osman I.H., "An introduction to Meta-Heuristics" (1995). Operational Research, Tutorial Papers, pp: 92-122

[35] Pierrreval H., Caux C., Paris J.L. \& Viguier F. (2003). Evolutionary approaches to the design and organization of manufacturing systems, Computers \& Industrial Engineering, Vol. 44, pp. 39-364.

[36] Sadrzadeh A., (2012). A genetic algorithm with the heuristic procedure to solve the multiline layout problem, Computers \& Industrial Engineering 62 1055-1064.

[37] Seehof J. M., \& Evans W. O., (1967). Automated layout design program, The Journal of Industrial Engineering, Vol. 18, pp. 690-695.

[38] Solimanpur M. \& Kamran M. A. (2010). Solving facilities location problem in the presence of alternative processing routes using a genetic algorithm, Computers \& Industrial Engineering 59 830-839.

[39] Taha H.A. (2012). Investigación de operaciones, Ed. Pearson Educación, México.

[40] Tam K.Y. (1992). A simulated Annealing for Allocation Space to Manufacturing Cells,
European Journal of Operational Research, Vol. 63, pp. 332-346.

[41] Tam K.Y. (1992). Genetic Algorithms, Function Optimization, and Facility Layout Design,International Journal of Production Research, Vol. 30, pp. 63-87.

[42] Tate, D.M. \& Smith A.E. (1995). Unequal Area Facility Layout Using Genetic Search, IEE Transactions, Vol. 27, pp. 465-472.

[43] Tompkins, J. A., White, J. A., Bozer, Y. A., Frazelle, E. H., Tanchoco, J. M. \& Trevino, J. (1996). Facilities planning, John Wiley, New York.

[44] Tong, X., (1991). SECOT: A sequential construction technique for facility design, Pittsburgh, PA, University of Pittsburgh, Doctoral Dissertation.

[45] Wong K.Y. \& Komarudin (2010). Solving facility layout problems using flexible bay structure representation and ant system algorithm, Expert System with Applications, 37, 55235527.

[46] Wu Y. \& Ji Ping (2008). Solving the Quadratic Assignment Problems by a Genetic Algorithm with New Replacement Strategy, International Journal of Information and Mathematical Sciences, 4:3.

[47] Xu G. \& Papageorgiou L.G. (2009). Process plant layout using an improvement-type algorithm, Chemical Engineering Research and Design, 87 780-788.

[48] Yang T., Peters B. A., \& Tu M. (2005). Layout design for flexible manufacturing systems considering single-loop directional flow patterns, European Journal of Operational Research, Vol. 164, N. pp. 440-455.

[49] Yongzhong Wu, and Ping Ji (2008). Solving the Quadratic Assignment Problems by a Genetic Algorithm with a New Replacement Strategy, International Journal of Information and Mathematical Sciences 4:3.

[50] Zanakis S.H. y Evans, J.R. (1981). "Heuristic optimization: Why, when and how to use it", Interfaces, 5.

[51] Facility Location and Layout Copyright (C) YihLong Chang 2000

[52] Burkard R. E., Cela E., Pardalos P. M. \& Pitsoulis L. S., (1998), Handbook of Combinatorial Optimization, Kluwer Academic Publishers, P. Pardalos and D.-Z. Du, eds. 